\title{
Relationship between anthropometry, body composition and blood pressure in apparently healthy males
}

\author{
S. M. P. Kerr, M. B. E. Livingstone, L. K. Forsythe and J. M. W. Wallace \\ Northern Ireland Centre For Food and Health, University of Ulster, Coleraine BT52 1SA, UK
}

Hypertension is diagnosed as a blood pressure (BP) reading of $140 / 90 \mathrm{mmHg}$ or higher and can play a causal role in adverse health consequences such as arterial damage and can lead to heart attack or stroke. There is a higher risk of hypertension among obese individuals compared to their lean counterparts ${ }^{(1)}$. Overall, recent research has concluded that a high systolic BP (SBP) is a greater risk factor than a high diastolic BP (DBP) for such health problems and it has been proposed that the assessment of DBP could be discontinued $^{(2)}$. It has been suggested that waist circumference (WC) correlates better with visceral adiposity and is a stronger predictor of hypertension and associated risk factors than $\mathrm{BMI}^{(3)}$. The aim of this study was to investigate associations between anthropometry, body composition and BP in healthy young males. Weight, height and WC were measured using standard procedures in 98 apparently healthy men (18-30 years) and BMI was calculated. BP was measured in duplicate (after firstly determining the reference arm) and the mean of the two readings calculated. Fat mass was determined via air displacement plethysmography using a calibrated BOD POD ${ }^{\circledR}$ (Life Measurement Inc.). Trunk fat was assessed using Dual X-ray absorptiometry (DXA) (Lunar Prodigy Pro ${ }^{\mathrm{TM}}$ ) and abdominal fat was determined using an abdominal fat analyser (Tanita Viscan visceral and trunk fat analyser).

\begin{tabular}{lllllll}
\hline & \multicolumn{3}{c}{ SBP } & & \multicolumn{2}{c}{ DBP } \\
\cline { 2 - 3 } & $\beta$ & $95 \%$ CI & $P^{*}$ & & $\beta$ & $95 \%$ CI \\
\hline Weight $(\mathrm{kg})$ & 0.09 & $(-7.78,20.35)$ & 0.38 & 0.20 & $(-0.51,18.70)$ & $P^{*}$ \\
BMI $\left(\mathrm{kg} / \mathrm{m}^{2}\right)$ & 0.11 & $(-0.27,0.91)$ & 0.28 & 0.22 & $(0.04,0.84)$ & 0.06 \\
WC $(\mathrm{cm})$ & 0.17 & $(-3.97,40.19)$ & 0.11 & 0.25 & $(3.19,33.31)$ & 0.84 \\
Fat mass $(\mathrm{kg})$ & 0.14 & $(-1.18,5.94)$ & 0.19 & 0.32 & $(1.46,6.18)$ & $<0.02$ \\
DXA trunk fat $(\%)$ & 0.23 & $(0.77,10.33)$ & 0.02 & 0.39 & $(3.03,9.35)$ & $<0.001$ \\
Abdominal fat $(\%)$ & 0.20 & $(-0.02,11.90)$ & 0.05 & 0.39 & $(3.86,11.67)$ & $<0.001$ \\
Visceral fat & 0.19 & $(-0.36,7.85)$ & 0.07 & 0.31 & $(1.44,6.96)$ & $<0.001$ \\
\hline
\end{tabular}

Positively associated with BP $(P<0.05$, multiple regression) including age as a covariate in each model.

In the group, the mean with their SD BMI (mean 24.91, SD 4.06 kg/m²), WC (mean 85.10, SD 9.50 cm), Fat Mass (mean 14.10, SD 9.29), Trunk Fat (\%) (mean 18.65, SD 7.51), SBP (mean 126.17, SD $11.83 \mathrm{mmHg}$ ) and DBP (mean 80.06, SD $7.97 \mathrm{mmHg}$ ).

The results showed that DXA trunk fat, abdominal fat and visceral fat were the only predictors of SBP. WC was a better predictor of DBP than BMI. DXA trunk fat, abdominal fat and visceral fat had a greater association with DBP than they did with SBP. As BMI and WC are the most widely utilised proxy measures of total and abdominal obesity, respectively, it can be concluded from the current results that WC is a stronger predictor of increased DBP than BMI. Therefore, when it is not feasible to measure trunk, abdominal or visceral fat directly, WC is the most appropriate measure to use as a predictor of DBP.

This work was funded by the Department for Employment and Learning. Ethical Approval was obtained from the University of Ulster Research Ethics Committee.

1. Haslam DW \& James WPT (2005) Obesity 366, 1197-1209.

2. Kannel WB (2000) Elevated systolic blood pressure as a cardiovascular risk factor. Am J Cardiol 85, 251-255.

3. Balkau B et al. (2007) International day for the evaluation of abdominal obesity (IDEA): a study of waist circumference, cardiovascular disease, and diabetes mellitus in 168000 primary care patients in 63 countries. Circulation 116, 1942-1951 